\title{
ANÁLISIS BIOARQUEOLÓGICO EN EL SITIO LOTEO SILVA. NUEVOS APORTES PARA EL VALLE INFERIOR DEL RÍO NEGRO (VIEDMA, PROVINCIA DE RÍO NEGRO)
}

\author{
GUSTAVO FLENSBORGa ${ }^{a}$, ESTEBAN LA VALLE ${ }^{b}$, FRANCISCA BERÓNc ${ }^{c}$ \\ YANINA MOREY ${ }^{d}$, FEDERICO SCARTASCINI ${ }^{e} \&$ JIMENA ALBERTI ${ }^{\dagger}$
}

\section{RESUMEN}

Se presenta el análisis bioarqueológico de los restos humanos rescatados en el sitio Loteo Silva (Viedma, provincia de Río Negro) con el objetivo de caracterizar y evaluar las prácticas funerarias, la funcionalidad del sitio, la paleodieta, y discutir la cronología de las ocupaciones humanas de cazadoresrecolectores en el curso inferior del río Negro. Asimismo, se discute e inserta este contexto en la arqueología del área de estudio y de microregiones aledañas (e.g., transición pampeano-patagónica oriental, costa norte del Golfo San Matías). Los resultados indican la presencia de tres individuos adultos de ambos sexos, inhumados tanto en modalidades primarias como secundarias simples. Los estudios tafonómicos dan cuenta de la estabilidad en la historia post-depositacional y la exposición de los restos en momentos cercanos a su recuperación. Análisis de isótopos estables muestran una dieta basada en recursos preferentemente terrestres. Dos fechados radiocarbónicos arrojaron una edad correspondiente al lapso ca. 3.600-3.200 años AP, indicando la ocupación más temprana conocida hasta el momento para el curso inferior del río Negro y la evidencia de manipulación antrópica de cadáveres más temprana del noreste de Patagonia. Los entierros recuperados en el sitio Loteo Silva presentan tendencias y características similares a otros contextos mortuorios del área de estudio y de regiones vecinas.

PALABRAS CLAVE: bioarqueología, rescate arqueológico, cazadores-recolectores, Holoceno medio final, noreste de Patagonia.

a Instituto de Investigaciones Arqueológicas y Paleontológicas del Cuaternario Pampeano (INCUAPA-CONICET), Facultad de Ciencias Sociales, Universidad Nacional del Centro de la Provincia de Buenos Aires (UNICEN), Argentina, Avenida del Valle 5737 (7400), Olavarría, Buenos Aires. $\measuredangle$ gflensbo@soc.unicen.edu.ar

b Departamento de Arqueología, Facultad de Ciencias Sociales, Universidad Nacional del Centro de la Provincia de Buenos Aires (UNICEN), Argentina, Avenida del Valle 5737 (7400), Olavarría, Buenos Aires. estebanlavalle1@gmail.com

c Departamento de Arqueología, Facultad de Ciencias Sociales, Universidad Nacional del Centro de la Provincia de Buenos Aires (UNICEN), Argentina, Avenida del Valle 5737 (7400), Olavarría, Buenos Aires. franciscaberon@gmail.com

d Departamento de Arqueología, Facultad de Ciencias Sociales, Universidad Nacional del Centro de la Provincia de Buenos Aires (UNICEN), Argentina, Avenida del Valle 5737 (7400), Olavarría, Buenos Aires. moreyyanina@gmail.com

e IIDyPCa-CONICET-Universidad Nacional de Río Negro, Bartolomé Mitre $6305^{\circ} \mathrm{B}$ (CPR8400AHL), San Carlos de Bariloche, Río Negro, Argentina. fscartascini@gmail.com

f Consejo Nacional de Investigaciones Científicas y Técnicas, Instituto Multidisciplinario de Historia y Ciencias Humanas (IMHICIHU-CONICET). Saavedra 15, 5to piso, (C1083ACA), Buenos Aires. jimealberti@gmail.com 


\title{
BIOARCHAEOLOGICAL ANALYSIS AT LOTEO SILVA SITE. NEW CONTRIBUTIONS FOR THE LOWER VALLEY OF THE NEGRO RIVER (VIEDMA, RIO NEGRO PROVINCE)
}

\begin{abstract}
The bioarchaeological analysis of the human remains rescued at Loteo Silva site (Viedma, Río Negro province) is presented, with the objective of characterizing and evaluating funerary practices, site functionality, paleodiet, and discussing the chronology of hunter-gatherer occupations from the lower course of the Negro River. In addition, this context is discussed and inserted in the archaeology of the study area and surrounding microregions (e.g., eastern Pampa-Patagonia transition, northern coast of San Matías Gulf). The results indicate the presence of three adult individuals of both sexes, buried in both simple primary and secondary modalities. The taphonomic studies show the stability in the post-depositional history and the exposure of the skeletons at moments close to their actual recovery. Stable isotope analysis indicates that mainly terrestrial resources were consumed by these individuals. Two radiocarbon dates showed a chronology of $c a$. 3.600-3.200 years BP, indicating the earliest occupation for the lower course of the Negro River and the earliest evidence of burial manipulation from the Northeastern Patagonia. The burials recovered at Loteo Silva site presents trends and characteristics similar to other mortuary contexts of the study area and neighboring regions.
\end{abstract}

KEY WORDS: Bioarchaeology, archaeological rescue, hunter-gatherers, final Middle Holocene, Northeastern Patagonia.

\section{INTRODUCCIÓN}

Los restos humanos constituyen uno de los registros arqueológicos más numerosos, destacadoseimportantesenelnorestedePatagonia desde fines del siglo XIX hasta la actualidad. El hallazgo de sitios con concentraciones de entierros humanos ha sido objeto de numerosas investigaciones arqueológicas, bioantropológicas y bioarqueológicas con el propósito de estudiar el poblamiento humano de Patagonia, los mecanismos adaptativos desarrollados por los grupos cazadores-recolectores a su entorno socio-ambiental, modos de organización social, etc. (Imbelloni, 1924-1925; Bórmida, 19531954; Cocilovo \& Guichón, 1994; Sardi, 2002; Barrientos \& Pérez, 2004; Pérez, 2006; Bernal et al. 2007, 2008; Favier Dubois et al. 2009; Gordón, 2011; Prates \& Di Prado, 2013, entre muchos otros). Particularmente en el curso inferior del río Negro se detectaron varios sitios con cientos de entierros humanos sobre pequeñas elevaciones de dunas, cercanos a lagunas, meandros abandonados y al propio río (e.g., laguna del Juncal, Rancho del Indio Pascual, San Javier; Moreno, 1874; Bórmida,
1950; Moldes de Entraigas, 1983; Fisher \& Nacuzzi, 1992, entre otros; Fig. 1). La mayoría de los restos humanos constituye colecciones de museos y carece de un registro detallado de sus contextos de hallazgos. Desde fines del siglo XIX hasta mediados del siglo XX, en los sitios y las colecciones se hicieron descripciones generales de las modalidades de entierro (e.g., primarios, secundarios), de la asociación de los restos humanos con materiales culturales interpretados como parte del ajuar funerario (Moreno, 1874; Bórmida, 1950), sobre deformaciones culturales de los cráneos (Imbelloni, 1924-1925; Bórmida, 1953-1954), y de las características funcionales de los sitios (e.g., cementerios, paraderostalleres; ver síntesis en Fisher \& Nacuzzi, 1992; Prates \& Di Prado, 2013). Posteriormente, desde fines del siglo XX y principios del siglo XXI, las investigaciones se centraron principalmente en el estudio de cráneos procedentes de colecciones de museos, para discutir temas relacionados con las prácticas culturales en la deformación cultural de esta unidad anatómica (Pérez et al. 2009; Serna et al. 2018), el poblamiento de Patagonia (Cocilovo \& Guichón, 1994; Sardi, 2002; González-José, 2003; Barrientos \& Pérez, 


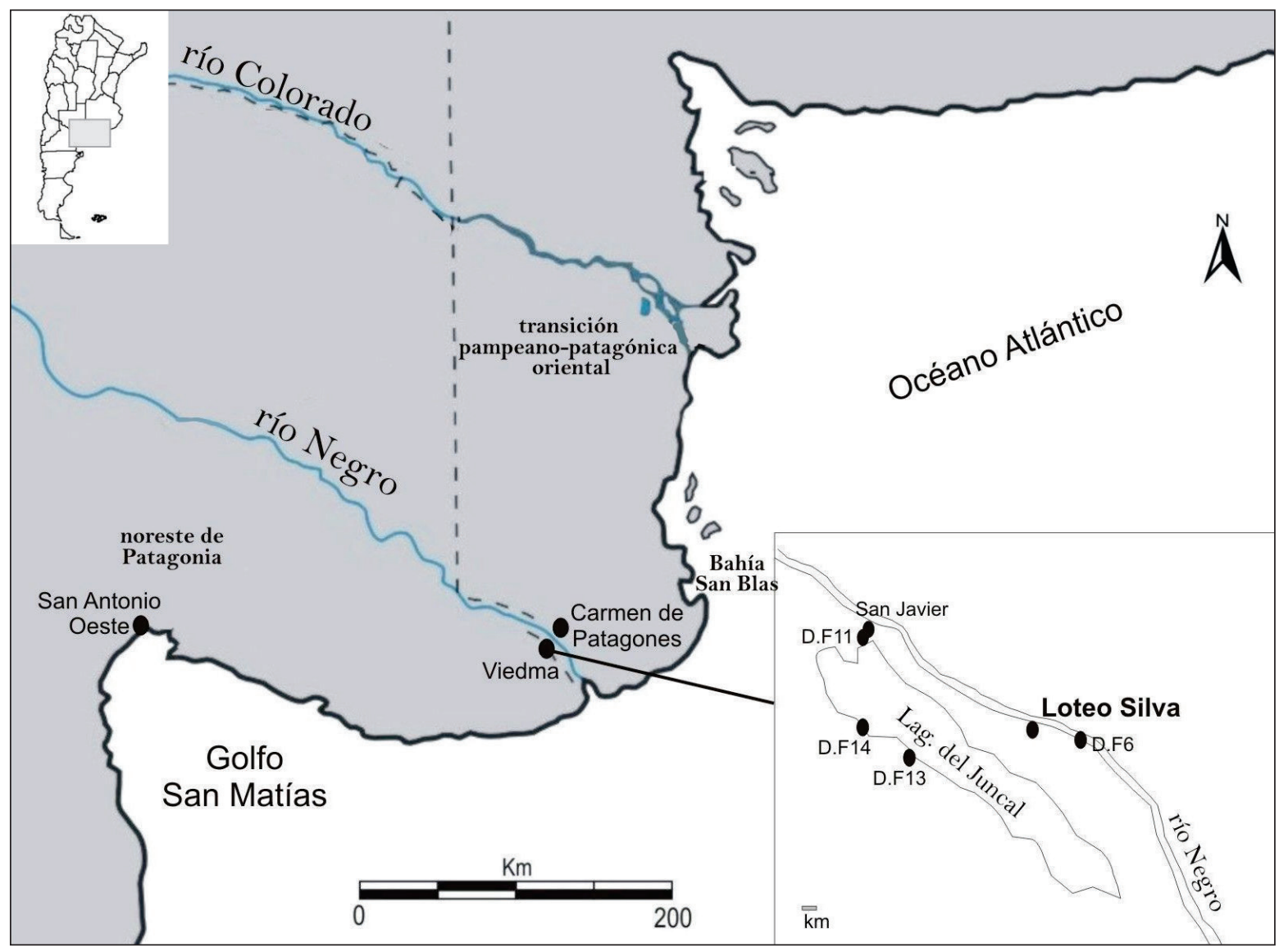

Fig. 1. Localización del sitio Loteo Silva en el curso inferior del río Negro, en relación a otros contextos arqueológicos con presencia de entierros humanos (Tomado y modificado de Fisher \& Nacuzzi, 1992). Nota: D.F: Distrito Federal.

2004), violencia interpersonal (Gordón, 2011), salud bucal (Bernal et al. 2007; Menéndez, 2010), cronología (Bernal et al. 2008), entre otros tópicos.

Como se observa en este escenario, desde hace décadas no se realizan excavaciones arqueológicas en el valle inferior del río Negro, hasta la presencia de un nuevo contexto funerario denominado Loteo Silva (Fig. 1), recuperado recientemente en una situación de rescate. El objetivo de este trabajo es presentar información bioarqueológica del sitio que permita caracterizar y evaluar las prácticas funerarias, la funcionalidad del sitio, la paleodieta, y discutir la cronología de las ocupaciones humanas de cazadores-recolectores en el curso inferior del río Negro. Finalmente se discute e inserta este caso de estudio en las investigaciones bioarqueológicas del noreste de Patagonia.

\section{CARACTERÍSTICAS DEL CONTEXTO ARQUEOLÓGICO}

El sitio arqueológico denominado Loteo Silva se ubica en uno de los barrios periféricos de la ciudad de Viedma $\left(40^{\circ} 49^{\prime} 32.9^{\prime \prime} \mathrm{S}, 63^{\circ} 00^{\prime}\right.$ 57.3" O). Durante la excavación de una zanja por parte de una empresa suministradora de gas en 2011 se encontraron elementos óseos humanos, los cuales fueron entregados para su custodia a la policía local. En el mes de agosto de dicho año, dos de los autores de este trabajo (F. S. y J. A.) fueron convocados para la recuperación de los entierros, pero las tareas de rescate (e.g., recolección de huesos superficiales, excavación) se interrumpieron debido a un desacuerdo entre los representantes de las comunidades originarias y la Secretaría de Cultura de la Provincia de Río Negro. En el mes de diciembre de 2011, se retomaron las actividades 


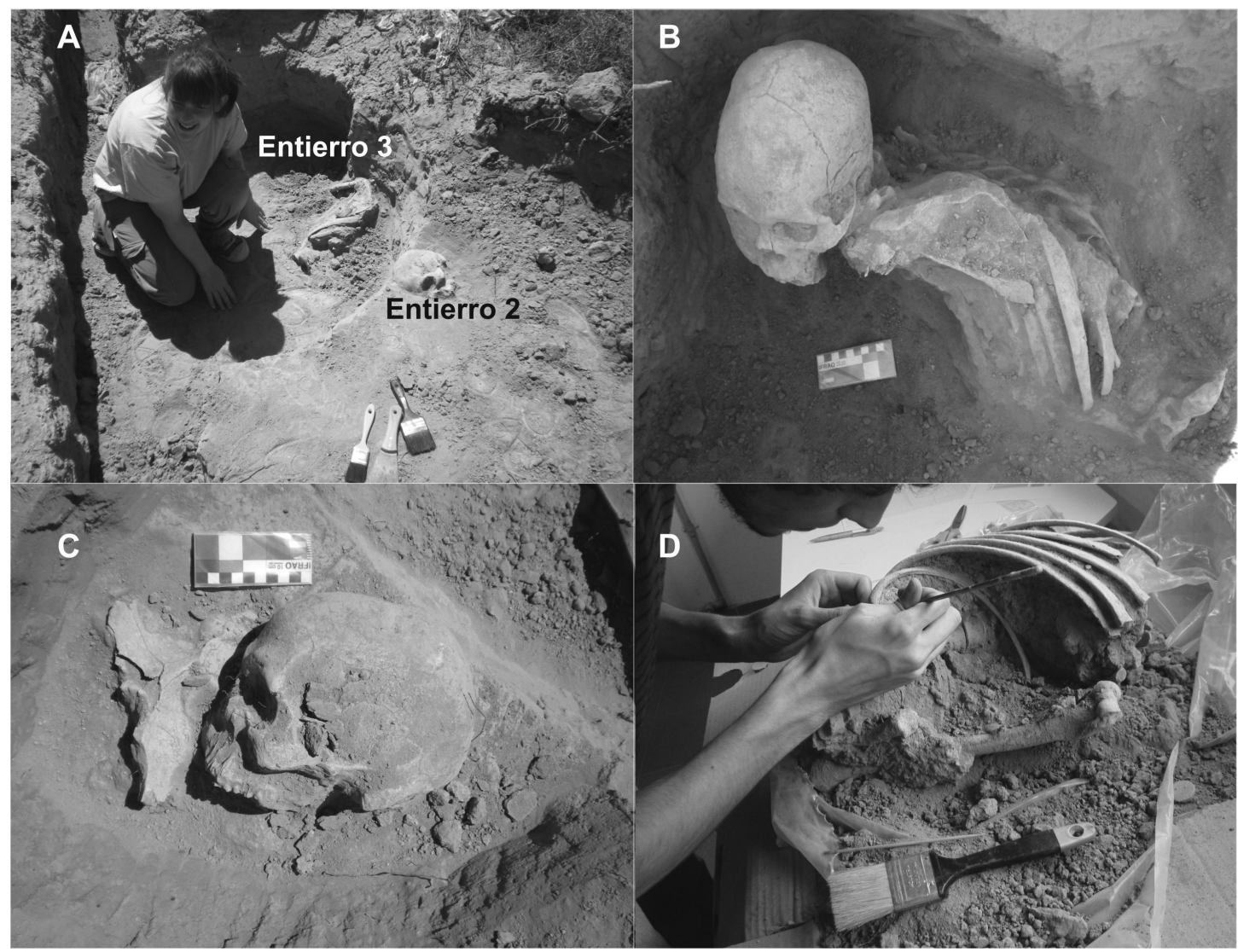

Fig. 2. Características del sitio Loteo Silva y del contexto de excavación. A) Ubicación de los Entierros 2 y 3 , espacialmente cercanos entre sí, a escasos centímetros de la superficie del suelo. B) Unidades anatómicas articuladas del Entierro 3 indicando la modalidad primaria. C) Cráneo y coxal del Entierro 2 asociado espacialmente, correspondiente a una modalidad secundaria simple. D) Excavación del Entierro 3 en el laboratorio de INCUAPA-CONICET.

en el sitio y se realizaron excavaciones para recuperar los restos humanos que permanecían semienterrados en el lugar (Fig. 2A). El contexto general del sitio se encontraba muy impactado por la remoción y la caída de sedimento, la presencia de basura actual y las tareas de instalación del caño de gas. Aun así, se hallaron in situ tres entierros muy cercanos espacialmente entre sí y a una profundidad de ca. $40 \mathrm{~cm}$ de la superficie del suelo. En los Entierros 1 y 3 las unidades anatómicas estaban articuladas (Fig. 2B), mientras que en el Entierro 2 se hallaron huesos desarticulados, asociados espacialmente (Fig. 2C). En el caso del Entierro 3, se extrajo en el sitio el sector postcraneal en forma de bloque y luego fue excavado (año 2016; Fig. 2D) en el laboratorio del Instituto de Investigaciones Arqueológicas y Paleontológicas del Cuaternario Pampeano (INCUAPA-CONICET).
Con posterioridad a las tareas de rescate, se realizó un breve informe sobre los trabajos realizados y las principales características de las muestras, que fue elevado a la Secretaria de Cultura de la Provincia de Río Negro, a las comunidades locales y a miembros del Consejo de Desarrollo de las Comunidades Indígenas (CODECI) (Scartascini \& Alberti, 2011). En principio, este conjunto de restos humanos fue custodiado por la Secretaría de Cultura de la Provincia de Río Negro, en el museo provincial Eugenio Tello, y posteriormente entregado a los Dres. Borella y Favier Dubois con el rótulo Acta $N^{\circ} 102$ (4/9/2011). Actualmente se encuentra depositado en el laboratorio de INCUAPA-CONICET, donde fue estudiado y serán devuelto a la autoridad de aplicación de la provincia de Río Negro por disposición de la Ley Nacional 25.743/03. 


\section{MÉTODOS}

Para el análisis cuantitativo de las partes esqueletarias se tomaron en cuenta el número de especímenes óseos (NISP), número mínimo de elementos (NME) y número mínimo de individuos (NMI) (Grayson, 1984; Klein \& Cruz-Uribe, 1984; Lyman, 1994). Se realizaron dos tipos de ensamblaje, uno mecánico y otro anatómico. El mecánico consistió en remontar especimenes fracturados que pertenecían a un mismo elemento, mientras que el anatómico se realizó sobre la base de la correspondencia bilateral y la unión intermembral (Todd \& Frison, 1992).

La determinación del sexo se realizó a partir de observaciones macroscópicas sobre diferentes rasgos morfológicos compilados por Buikstra y Ubelaker (1994) en elementos óseos correspondientes a individuos adultos. En este sentido, se analizaron rasgos del cráneo y de la mandíbula (e.g., protuberancia externa del occipital, glabela, procesos mastoideos, arcos supraorbitarios y proyección de la eminencia mentoniana; Buikstra y Ubelaker, 1994). En los coxales se tuvieron en cuenta los rasgos y las dimensiones de la escotadura ciática mayor, el criterio de arco y la morfología de la región subpúbica (concavidad subpúbica, superficie medial y arco ventral) (Phenice, 1969; Bruzek, 2002; Barboza et al. 2004). Finalmente, para el sacro se consideró la morfología de la superficie auricular y las dimensiones del mismo (ancho y largo; Krogman, 1962; Flandes, 1978).

La edad de muerte en individuos adultos se estimó teniendo en cuenta el estado de fusión de las suturas craneanas (Buikstra \& Ubelaker, 1994), así como de las epífisis de algunos huesos que maduran durante la etapa de adulto joven (e.g., clavículas, vértebras dorsales y lumbares; Owings Webb \& Suchey, 1985; Albert \& Maples, 1995; Albert et al. 2010). En los coxales se analizaron los cambios morfológicos presentes en la superficie auricular (Buckberry y Chamberlain, 2002) y en la sínfisis púbica (Brooks y Suchey, 1990).

Con el propósito de estudiar los agentes y procesos tafonómicos que intervinieron en el registro bioarqueológico se realizó un análisis macroscópico de la superficie de los huesos. Se consideraron las siguientes variables: meteorización, carbonato de calcio $\left(\mathrm{C}_{\mathrm{a}} \mathrm{CO}_{3}\right)$, dióxido de manganeso
$\left(\mathrm{MnO}_{2}\right)$, marcas de roedores, de carnivoros y de raíces (Behrensmeyer, 1978; Binford, 1981; Lyman \& Fox, 1989; Lyman, 1994; Gutiérrez, 2004; González, 2012). Para estimar el grado de integridad macroestructural de la unidad ósea, se calculó el porcentaje de hueso presente respecto del total esperado. Se establecieron cuatro rangos para describir la completitud del hueso, las marcas de raíces, el carbonato de calcio y el dióxido de manganeso: $<25 \%, 26-50 \%, 51-75 \%$ y $76-$ $100 \%$ (González, 2012). Finalmente, se evaluó de manera macroscópica la presencia de tinciones rojizas y huellas de corte y de manera morfoscópica la deformación cultural del cráneo (Dembo \& Imbelloni, 1938). Dos fechados radiocarbónicos fueron realizados en el Laboratorio AMS de la Universidad de Arizona y los valores de isótopos estables $\left(\delta^{13} \mathrm{C}\right.$ y $\left.\delta^{15} \mathrm{~N}\right)$ en el Departamento de Geociencia de la Universidad de Arizona.

\section{RESULTADOS}

En el sitio Loteo Silva se recuperó un número mínimo de tres individuos adultos correspondientes a ambos sexos (Tabla 1). El Entierro 1 está representado por un individuo femenino de entre 20-23 años y conformado por 26 unidades anatómicas, principalmente por cráneo, mandíbula, huesos largos y cinturas escapulares y pélvicas (Fig. 3). En base a la articulación que presentaron las unidades anatómicas durante su recuperación, se infiere que se trata de un entierro primario simple, aunque no fue posible determinar la posición del mismo. El cráneo se encontraba muy fragmentado para evaluar la deformación cultural.

El Entierro 2 está representado por un individuo masculino de entre 25-40 años y constituido por dos elementos óseos, un cráneo y un coxal (Figs. 2A y 3). La relación espacial de los dos elementos óseos, es decir, el cráneo asociado y semi-yuxtapuesto al coxal (Fig. 2C), indicaría que se trata de una modalidad secundaria simple (ver discusión). No se observó deformación cultural del cráneo (Fig. 4).

El Entierro 3 está representado por un individuo probablemente masculino de entre 3550 años y conformado por 53 elementos óseos, principalmente por vértebras, costillas, cráneo, mandíbula y coxal (Fig. 3). En base a la articulación 
Tabla 1. Cronología, isótopos estables e información bioarqueológica de los individuos del sitio Loteo Silva. Nota: P.: probablemente, NISP: número de especímenes óseos; NME: número mínimo de elementos.

\begin{tabular}{ccccccccc}
\hline Entierro & $\begin{array}{c}\text { Modalidad } \\
\text { entierro }\end{array}$ & Sexo & $\begin{array}{c}\text { Edad } \\
\text { (años) }\end{array}$ & NISP & NME & $\begin{array}{c}\mathrm{C}^{14} \\
\text { años AP }\end{array}$ & $\delta^{13} \mathrm{C}$ & $\delta^{15} \mathrm{~N}$ \\
\hline 1 & primario & femenino & $20-23$ & 43 & 26 & - & - & - \\
2 & secundario & masculino & $25-40$ & 18 & 2 & $3.247 \pm 27$ & $-20 \%$ o & $11.5 \%$ o \\
3 & primario & P. masculino & $35-50$ & 88 & 53 & $3.598 \pm 30$ & $-19.6 \%$ o & $11.7 \%$ 。 \\
\hline Total & - & - & - & 149 & 81 & & & \\
\hline
\end{tabular}

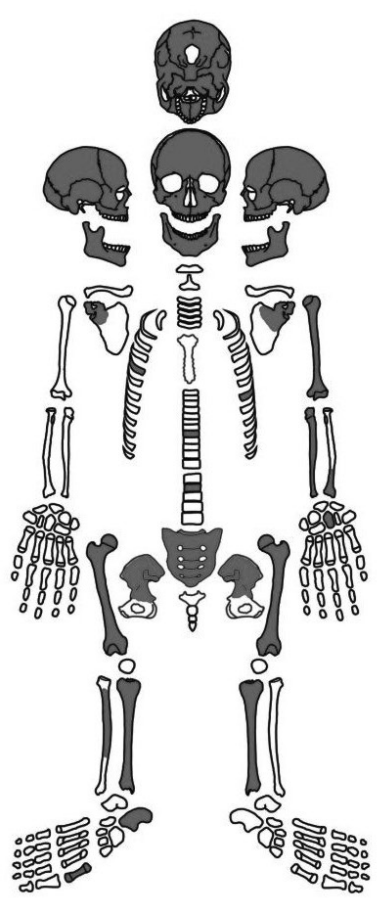

Entierro 1

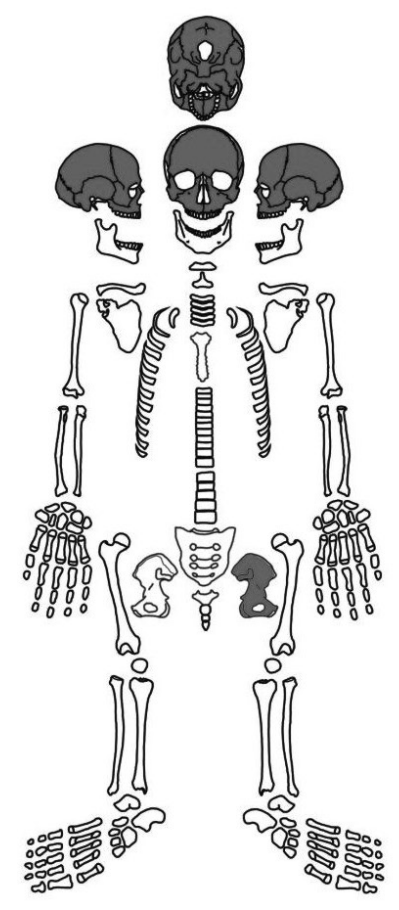

Entierro 2

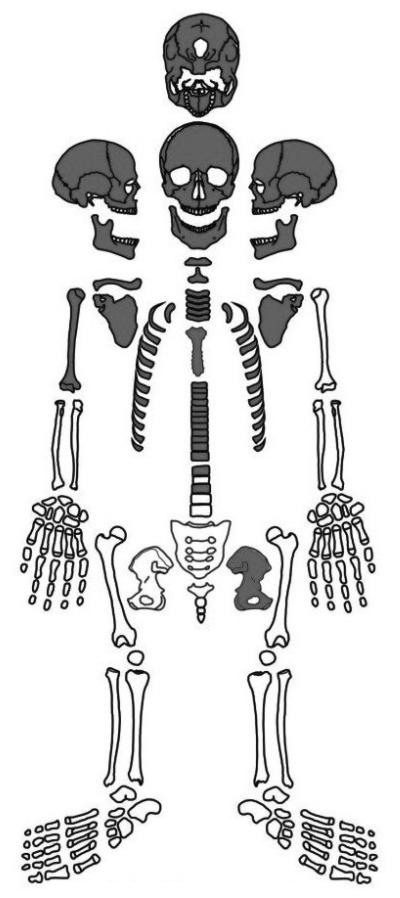

Entierro 3

Fig. 3. Representación de partes esqueletarias en el sitio Loteo Silva.

de todos los huesos presentes y la posición del entierro (Fig. 2D), se infiere que se trata de un primario simple en posición decúbito lateral derecho. No se observó deformación cultural del cráneo (Fig. 4).

Desde el punto de vista tafonómico se observó una baja representación de las variables estudiadas en los tres entierros. En este sentido, no se documentó meteorización ni marcas de carnivoros. Teniendo en cuenta todos los huesos en su conjunto, se registró baja frecuencia de marcas de roedores (1,23\%; 1/81; sólo en el Entierro 3). Además se observó un bajo porcentaje de $\mathrm{C}_{\mathrm{a}} \mathrm{CO}_{3}$ (8,64\%; 7/81; sólo en el Entierro 1), a diferencia del $\mathrm{MnO}_{2}$ que se encuentra en alta frecuencia $(85,18 \%$; 69/81) y en todos los individuos, aunque la tinción afectó a un bajo porcentaje de la superficie cortical (0-50\%). La variable tafonómica más representada en los tres individuos es la acción de las raíces $(87,65 \% ; 71 / 81)$, aunque sólo alteró los intervalos comprendidos entre $0-50 \%$ de la superficie ósea. Respecto de la completitud ósea, 


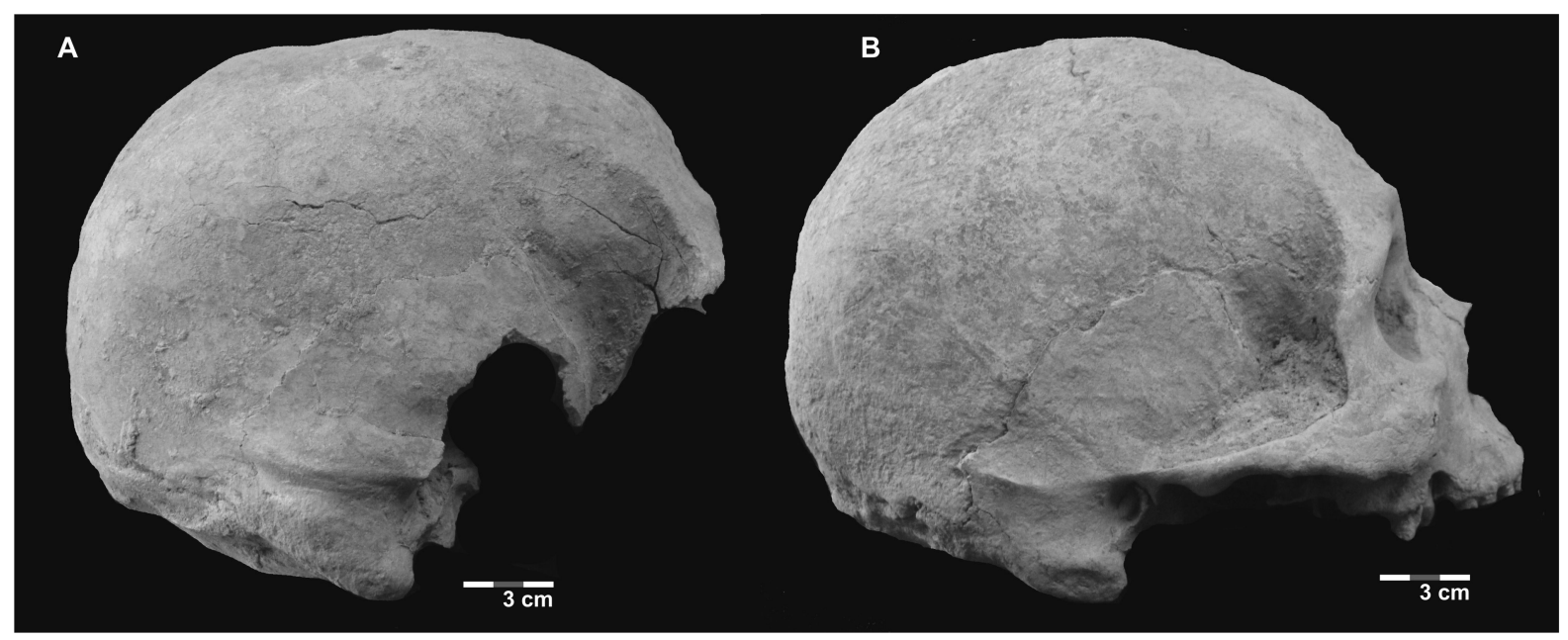

Fig. 4. Morfología de la bóveda de los cráneos. A) Entierro 2. B) Entierro 3.

el $71,6 \%$ de los huesos se encuentra en el rango más elevado (76-100\%), mientras que el resto se encuentra comprendido dentro del intervalo $51-75 \%$. No se detectaron huellas de corte en el conjunto óseo. La presencia de una tenue tinción rojiza se observó en el parietal y temporal izquierdo del cráneo perteneciente al Entierro 3.

Se realizaron dos fechados radiocarbónicos en diferentes individuos (Tabla 1). Se seleccionó un fragmento de cráneo del Entierro 2 que arrojó un valor de $3.247 \pm 27$ años AP (AA-111525; 3.359-3.550 años cal AP) y un fragmento de costilla del Entierro 3 que indicó un valor de 3.598 \pm 30 años AP (AA110574; 3.719-3.966 años cal $\mathrm{AP})$. Asimismo, el análisis de isótopos estables dio cuenta de valores de $\delta^{13} \mathrm{C}=-20 \%$ y $\delta^{15} \mathrm{~N}=11.5 \%$ 。 para el Entierro 2 y $\delta^{13} \mathrm{C}=-19.6 \%$ o y $\delta^{15} \mathrm{~N}=11.7 \%$ 。 para el Entierro 3. La relación $\mathrm{C} / \mathrm{N}$ es de 3.2 en ambos casos y se ubica dentro del rango aceptado para muestras sin alteración diagenética (DeNiro, 1985).

\section{DISCUSIÓN}

En el sitio Loteo Silva se pudieron recuperar tres esqueletos en un contexto perturbado por obras de construcción. A pesar de esto, los trabajos de rescate y los estudios de laboratorio permitieron realizar un análisis bioarqueológico detallado. De acuerdo con lo expresado en apartados anteriores, al menos dos entierros (E2 y E3) no habrian sido afectados directamente por las obras (Fig. 2), mientras que en un caso (E1) se produjo la remoción del entierro sin intervención arqueológica.

Los resultados del estudio tafonómico en los tres individuos indican estabilidad en la historia de formación del sitio. La falta de evidencia de meteorización sugiere que los huesos no estuvieron expuestos en superficie o, por lo menos, no el tiempo suficiente como para meteorizarse (Behrensmeyer, 1978). Esta baja exposición en superficie se sustenta, además, en la ausencia de actividad de carnívoros (e.g., zorros, comadrejas, etc.) sobre los restos óseos (Binford, 1981; Lyman \& Fox, 1989; Martin, 2002). Las marcas de raíces y la tinción de dióxido de manganeso sugieren que los restos humanos estuvieron enterrados. El alto porcentaje de marcas de raíces sobre la superficie cortical es esperable debido a la poca profundidad a la que se hallaron enterrados los esqueletos respecto de la superficie del suelo, donde se encuentra la vegetación actual. La presencia de tinción de dióxido de manganeso en forma de manchas puntiformes es indicadora de focos de actividad de microorganismos durante la fase de diagénesis temprana (Bratina et al. 1998), aunque no se descarta el contacto de los huesos con suelos anegados o con eventuales ciclos de humedad, probablemente vinculada a depositaciones húmedas, palustres y/o fluviales (Gifford-González, 1981; López-González et al. 2006; González, 2007). Esto último está en sintonía con la precipitación de $\mathrm{C}_{2} \mathrm{CO}_{3}$ en algunos huesos del Entierro 1, ya que esta variable se manifiesta 
con frecuencia como producto de diversos procesos como la lixiviación, la pedogénesis y la diagénesis, vinculados a las oscilaciones del nivel freático (Daniels, 1981; Gutiérrez, 2004; González, 2007). Estas últimas dos variables tafonómicas indican que los huesos enterrados estuvieron expuestos a condiciones de humedad, muy probablemente debido a la cercanía del sitio al río Negro y/o a antiguas lagunas. De acuerdo con toda la información tafonómica, sumado a las características del contexto de hallazgo (e.g., asociación espacial entre entierros, articulación de unidades anatómicas), se propone que los individuos, aún con evidencia de manipulación antrópica (ver más adelante), fueron inhumados en momentos muy cercanos a la muerte y permanecieron enterrados hasta su recuperación actual.

En los tres esqueletos se observó un bajo porcentaje de supervivencia y representación diferencial de partes esqueletarias. Esto es interesante dado que si se asume una estabilidad post-depositacional y adecuada preservación del registro óseo, se debería esperar una mayor completitud esqueletal. En el caso del Entierro 1 esto podría ser explicado por la recuperación inicial de los huesos por parte de la policía local, antes de la llegada de los arqueólogos, lo que pudo generar una recolección sesgada de las unidades anatómicas. Pero en los dos entierros restantes, muy probablemente el factor de primer orden se corresponda con las modalidades de inhumación. Como se observa en la Fig. 2C, el Entierro 2 está conformado por dos unidades anatómicas desarticuladas. Aunque no se registraron huellas de corte, la ubicación del cráneo asociado a un coxal podría ser el resultado de la manipulación antrópica intencional de partes esqueletarias. Se descarta cualquier proceso post-depositacional de origen natural que pudiera generar el desplazamiento de unidades anatómicas en el sitio (e.g., actividades de roedores, acción hídrica). Además, desde una perspectiva comparativa, esta asociación espacial entre cráneos y coxales es frecuentemente registrada en las prácticas mortuorias del noreste de Patagonia, la transición pampeano-patagónica oriental, Subregión Pampa Seca y el sudeste de la región Pampeana para los últimos ca. 1.000 años AP (e.g., sitios La Petrona, Paso Alsina 1, Centro Minero, laguna Los Chilenos 1, Chenque
I; Barrientos et al. 2002; Flensborg et al. 2011, 2017; Mariano, 2011; Martínez et al. 2012; Berón, 2018). En el caso del Entierro 3 los huesos del cráneo, el tronco, la columna vertebral y la cintura escapular se hallaron completamente articulados. De acuerdo con los resultados tafonómicos, la ausencia de los miembros superiores e inferiores no podría ser explicada por la intervención de procesos naturales de formación de sitio. Por el contrario, se propone que factores culturales relacionados con la manipulación del individuo al momento del entierro o tiempo posterior al evento de inhumación podrían explicar la representación diferencial de partes esqueletarias. En este caso, si bien no se observaron huellas de corte, ni claras evidencias de manipulación de unidades anatómicas (e.g., desarticulación), la explicación más probable para la ausencia de ciertos sectores del esqueleto se correspondería con la selección intencional de partes anatómicas para el armado de paquetes funerarios. Esta situación también ha sido propuesta para otros contextos mortuorios de la transición pampeano-patagónica oriental (e.g., La PetronaEntierro 3; ca. 450 años AP; Flensborg et al. 2011) y Subregión Pampa Seca (e.g., Chenque I-Entierro 17; ca. 1.000 años AP; Berón, 2018). De acuerdo con lo expuesto, las modalidades de entierro registradas en Loteo Silva son variadas: primario simple (Entierro 1), secundario simple (Entierro 2) y primario simple, aunque probablemente con indicios de manipulación antrópica (Entierro 3). La asociación espacial de diferentes prácticas de inhumación en un mismo sitio también ha sido reportada desde fines del siglo XIX en otros contextos arqueológicos tanto del valle inferior del río Negro (Moreno, 1874; Bórmida, 1950; Fisher \& Nacuzzi, 1992), como del noreste de Patagonia (Bernal et al. 2008; Mariano, 2011; Prates \& Di Prado, 2013) y la transición pampeano-patagónica oriental durante el Holoceno tardío (Martínez et al. 2012; Flensborg et al. 2017).

Uno de los aportes más novedosos del sitio Loteo Silva a la arqueología local y del noreste de Patagonia está relacionado con la cronología obtenida en dos entierros. Respecto de las modalidades de inhumación, en el sitio se observan las evidencias más tempranas de manipulación de partes esqueletarias y presencia de entierros secundarios en la región, para una 
cronología de ca. 3.600-3.200 años AP. En este sentido, en la transición pampeano-patagónica oriental se observaron modalidades exclusivamente primarias entre los ca. 5.900-1.400 años AP, mientras que a partir de los ca. 1.400 años $\mathrm{AP}$ se comenzaron a realizar también entierros secundarios $y$, en algunos casos, asociados espacialmente con primarios (e.g., sitios Zoko Andi 1, La Petrona; Flensborg et al. 2017). En la costa norte del Golfo San Matías ocurre una situación similar, ya que entre los ca. 3.100-1.200 años AP, las modalidades fueron exclusivamente primarias (Favier Dubois et al. 2007; García Guraieb et al. 2010; Mariano, 2011), mientras que a partir de los ca. 1.200 años AP se comienzan a practicar entierros secundarios, en conjunto con los primarios (e.g., sitios Bajo de la Quinta-Cima de los Huesos; Mariano, 2011). De esta forma, las modalidades de entierro en Loteo Silva contribuyen a ampliar la variabilidad y complejidad de las prácticas mortuorias en el noreste de Patagonia para momentos más tempranos.

Hasta el momento las ocupaciones humanas en el valle inferior del río Negro se correspondían con el Holoceno tardío, a partir de los fechados radiocarbónicos obtenidos en restos humanos de la laguna del Juncal (ca. 3.100-400 años AP; Bernal et al. 2008) y en diferentes cráneos procedentes del valle inferior del río Negro (ca. 3.300-500 años AP; Gordón, 2011). Sin embargo, la edad cronológica obtenida en el Entierro 3 permite extender la ocupación del área hacia momentos más tempranos, indicando el uso del espacio para la inhumación de los difuntos a partir del Holoceno medio final. Este nuevo fechado se inserta en el esquema cronológico de los entierros humanos correspondientes al Holoceno medio del noreste de Patagonia y de la transición pampeanopatagónica oriental. La costa norte del Golfo San Matías cuenta con un individuo de ca. 4.800 años AP (sitio Bahía de San Antonio; Favier Dubois \& Scartascini, 2012), en las inmediaciones de Bahía San Blas se reportó un entierro en ca. 4.100 años AP (La Serranita, sitio C; Sanguinetti de Bórmida, 1999) y en el curso inferior del río Colorado se dieron a conocer varios individuos entre ca. 5.9004.100 años AP (sitios La Modesta, Loma de los Morteros, Tres Bonetes y Cantera de Rodados Villalonga; Martínez \& Flensborg, 2018).
La edad cronológica de ambos entierros coincide con el esquema clasificatorio generado a partir de la deformación craneana artificial propuesto para las regiones del sudeste de Pampa y noreste de Patagonia (Bernal et al. 2008; Berón \& Luna, 2009). En este sentido, es esperable que los cráneos del Entierro 2 y 3 no presenten deformación, ya que de acuerdo con la línea de tiempo definida por Berón y Luna (2009) respecto de la deformación tabular erecta y sus variantes, no hay registro de este rasgo para momentos previos a los ca. 3.150 años AP.

Respecto de la ocupación del sitio, ambos fechados radiocarbónicos dan cuenta del uso reiterado de este lugar para la inhumación de los individuos. Este escenario de reocupación o de ocupación múltiple de los mismos espacios es esperable para el noreste de Patagonia. Son numerosos los contextos mortuorios que indican el uso del paisaje exclusivamente para la inhumación y/o para funciones de campamentos base de actividades múltiples (incluyendo entierros humanos) a través de cientos o miles de años y que han sido definidos como lugares persistentes (ver discusión en Martínez et al. 2012; Prates \& Di Prado, 2013). Hay sectores del paisaje que tienen importancia sagrada para los grupos humanos, tal como puede ser el curso inferior del río Negro, ya que es un área que fue utilizada para la inhumación de cientos de individuos a lo largo de miles de años (e.g., laguna del Juncal; Figura 1; Bernal et al. 2008). Por lo tanto, Loteo Silva puede ser considerado como parte de este paisaje ritualizado y sacralizado por los grupos cazadoresrecolectores.

Un aspecto relevante en el sitio es que durante las tareas de excavación no se observaron materiales culturales asociados a los esqueletos, lo que indicaría que fueron inhumados sin objetos personales y/o ajuar funerario. Esta característica es compartida con varios sitios arqueológicos del área $y$ de regiones vecinas (e.g., curso medio e inferior del río Negro, costa del Golfo San Matías, transición pampeano-patagónica oriental; Bórmida, 1950; Mariano, 2011; Martínez et al. 2012; Prates \& Di Prado, 2013; Cardillo \& Borella, 2017). Aunque contrasta con algunos sitios observados en el curso inferior del río Negro (e.g., D.F.6; D.F.11, D.F.14; Fig. 1), ya que desde fines del siglo XIX se hace 
alusión a la presencia de numerosos esqueletos asociados a materiales culturales (e.g., puntas de proyectil, raspadores, molinos, fragmentos de cerámica y huesos de diferentes tipos de faunas; ver Lista, [1880] 1975; Moreno, 1874; Fisher \& Nacuzzi, 1992). Si bien en un principio esta asociación contextual fue interpretada como una derivación material de los acompañamientos funerarios en las sepulturas y de festines durante las ceremonias de entierro, actualmente es interpretada como parte del resultado de las actividades domésticas realizadas por los grupos cazadores-recolectores (ver discusión en Martínez, 2010; Prates \& Di Prado, 2013). Realizar una interpretación acerca de la funcionalidad del sitio parece un desafío, principalmente debido a que la superficie excavada es pequeña y no se realizaron excavaciones planificadas, ni el tamizado del sedimento extraído. Pero llama la atención que no se hayan recuperado restos de fauna y de materiales líticos cercanos a los entierros, correspondientes a ocupaciones domésticas, lo que permite proponer a modo hipotético que Loteo Silva se trataría de un área exclusiva de entierros humanos.

Los resultados de isótopos estables $\left(\delta^{13} \mathrm{C}\right.$ y $\delta^{15} \mathrm{~N}$ ) indican que los individuos se caracterizaron por una dieta basada principalmente en recursos terrestres. Por el momento no se han realizado estudios paleodietarios a través de esta vía de análisis en el curso inferior del río Negro, por lo que constituye un antecedente pionero en este aspecto. A nivel regional existe un esquema paleodietario más completo de los grupos cazadores-recolectores, a partir del estudio de restos humanos. En la costa norte del Golfo San Matías, se propone la ingesta de recursos marinos de elevado nivel trófico para el lapso 3.100-2.200 años AP y dietas mixtas a predominantemente terrestres de menor nivel trófico entre 1.500-420 años AP (Favier Dubois et al. 2009). Por su parte, en la transición pampeano-patagónica oriental los resultados de isótopos estables indican una dieta tanto de origen marino como terrestre para el Holoceno medio (ca. 6.000-4.100 años AP), mixta a preferentemente terrestre para el Holoceno tardío inicial (ca. 3.0001.000 años AP) y preferentemente terrestre para el Holoceno tardío final (ca. 1.000-250 años AP) (Flensborg et al. 2018). En este sentido, los resultados presentados aquí indican una situación diferente a la observada en regiones vecinas, ya que en estos dos individuos predominó la ingesta de herbívoros terrestres que se alimentaron de plantas de tipo $\mathrm{C}_{3}$ durante los ca. 3.600-3.200 años AP. No obstante esta información deberá ser discutida en futuros trabajos con una mayor cantidad de datos de isótopos estables procedentes del valle inferior del río Negro.

\section{CONCLUSIONES}

La intervención inmediata de arqueólogos en situaciones de rescate y el empleo de criterios metodológicos rigurosos son claves para salvaguardar parte del patrimonio cultural y para obtener datos valiosos que permitan estudiar $y$ comprender procesos sociales, aspectos culturales y modos de vida de grupos humanos del pasado, tanto a escala areal como regional (Serna \& Romano, 2018). En el caso del sitio Loteo Silva, la convocatoria de arqueólogos por parte de la Secretaria de Cultura de la Provincia de Río Negro para el rescate de los entierros humanos, permitió obtener un registro detallado del contexto y extraer los esqueletos con procedimientos adecuados para su preservación.

Las medidas adoptadas para la recuperación de los esqueletos permitieron obtener información desde el punto de vista cronológico, tafonómico, cuantitativo, paleodietario y de las prácticas mortuorias. En este sentido, en Loteo Silva se inhumaron individuos en modalidades primarias y secundarias simples, en una superficie acotada. La ausencia de materiales culturales podría indicar que se trata de un área exclusiva de entierros humanos. Los estudios paleodietarios, a través de isótopos estables, muestran dietas preferentemente terrestres. Finalmente, la cronología indica que se trata de la ocupación más temprana registrada para el valle inferior del río Negro, correspondiente a momentos finales del Holoceno medio y, además, presenta la evidencia de manipulación antrópica de cadáveres más temprana del noreste de Patagonia.

Este caso de estudio pretende ser un antecedente sobre el manejo y el estudio de restos humanos, así como de la coordinación y el trabajo conjunto de diferentes actores involucrados (comunidad científica, organismos gubernamentales, pueblos originarios y otros 
miembros de la sociedad local). Los datos aquí generados pueden ser utilizados por los distintos actores sociales, con el objetivo de construir una idea conjunta sobre el pasado cultural y social del área, y gestionar los modos más adecuados al momento de la detección, excavación, estudio, devolución de los restos humanos y su destino final.

\section{AGRADECIMIENTOS}

Al INCUAPA-CONICET por brindar las instalaciones y los equipos para llevar a cabo el análisis. A la Secretaría de Cultura de la provincia de Río Negro y al museo provincial Eugenio Tello. A la Dra. Borella y el Dr. Serna por los comentarios realizados a una versión previa. A dos revisores anónimos que permitieron mejorar el contenido del manuscrito. Las investigaciones fueron financiadas con un proyecto científico otorgado por la Agencia Nacional de Promoción Científica y Tecnológica (PICT 616-15).

\section{BIBLIOGRAFÍA}

Albert, A., \& Maples, W. (1995). Stages of epiphyseal union for thoracic and lumbar vertebral centra as a method of age determination for teenage and young adult skeletons. Journal of Forensic Sciences, 40(4), 623-633.

Albert, A., Mulhern, D., Torpey M. A. \& Boone, E. (2010). Age estimation using thoracic and first two lumbar vertebral ring epiphyseal union. Journal of Forensic Sciences, 55(2), 287-294.

Barboza, C., Bordach, M. A., \& Mendonça, O. (2004). Osteología Humana. Determinación de la edad y el sexo. El sitio SJ TIL 43. Río Cuarto: Universidad Nacional de Río Cuarto.

Barrientos, G., \& Pérez, S. I. (2004). La expansión y dispersión de poblaciones del norte de Patagonia durante el Holoceno tardío: evidencia arqueológica y modelo explicativo. En Civalero, T., Fernández, P. y Guraieb, G. (Eds.), Contra Viento y Marea. Arqueología de Patagonia, (pp. 179195). Buenos Aires: Instituto Nacional de Antropología y Pensamiento Latinoamericano y Sociedad Argentina de Antropología.

Barrientos, G., Oliva, F., \& del Papa, M. (2002). Historia pre $y$ postdepositacional del entierro secundario del sitio laguna los Chilenos 1 (provincia de Buenos Aires). Relaciones de la Sociedad Argentina de Antropología, XXVII, 303-325.
Behrensmeyer, A. K. (1978). Taphonomic and ecological information from bone weathering. Paleobiology, 4(2), 150-162.

Bernal, V., Novellino, P., González, P. \& Perez, S. I. (2007). Role of wild plant foods among Late Holocene huntergatherers from Central and North Patagonia (South America): an approach from dental evidence. American Journal of Physical Anthropology, 133, 1047-1059.

Bernal, V., González, P. N., Pérez, S. I., \& Pucciarelli H. (2008). Entierros humanos del nordeste de Patagonia: nuevos fechados radiocarbónicos. Magallania, 36(2), 175-183.

Berón, M. (2018). El sitio Chenque I. Un cementerio prehispánico en la Pampa Occidental. Estilo de vida $e$ interacciones culturales de cazadores-recolectores del Cono Sur Americano. Buenos Aires: Sociedad Argentina de Antropología.

Berón, M., \& L. H. Luna (2009). Distribución espacial y cronológica de la deformación craneana tabular erecta en Pampa y Norpatagonia. En M. Salemme, F. Santiago, M. Álvarez, E. Piana, M. Vázquez \& E. Mansur, E. (Eds.), Tendencias teórico metodológicas y casos de estudio en la Arqueología de la Patagonia, (pp 561-575). Ushuaia: Utopías.

Binford, L. R. (1981). Bones. Ancient Men and Modern Myths. New York: Academia Press.

Bórmida, M. (1950). Cementerios indígenas prehispánicos en la zona de la Laguna del Juncal. Anales del Museo Nahuel Huapi Perito Dr. Francisco P. Moreno, 2, 101-108.

Bórmida, M. (1953-1954). Los antiguos patagones. Estudio craneológico. Runa, 6, 1-95.

Bratina, B. J., Stevenson, B. S., Green, W. J., \& Schmidt, T. M. (1998). Manganese reduction by microbes from the oxic regions of the Lake Vanda (Antarctica) water column. Applied and Environmental Microbiology, 64, 3791-3797.

Brooks, S., \& Suchey, J. (1990). Skeletal age determination based on the Os Pubis: a comparison of the AcsadiNemeskeri and Suchey-Brooks methods. Human Evolution, 5, 227-238.

Bruzek, J. (2002). A Method for Visual Determination of Sex, Using the Human Hip Bone. American Journal of Physical Anthropology, 117, 157-168.

Buckberry, J. L., \& Chamberlain, A.T. (2002). Age estimation from the auricular surface of the ilium: A revised method. American Journal of Physical Anthropology, 119, 231-239.

Buikstra, J., \& Ubelaker, D. (1994). Standards for data 
collection from human skeletal remains: Proceedings of a Seminar at the Field Museum of Natural History. Arkansas Archaeological Survey Research Series, 44. Arkansas.

Cardillo, M., \& Borella, F. (2017). Caracterización métrica y morfológica de las cuentas provenientes del área costera norpatagónica. Río Negro, Argentina. Arqueología, 23(2), 81-97.

Cocilovo, J. A., \& Guichón, R. A. (1994). La deformación craneana "pseudocircular" en el grupo prehistórico de Laguna del Juncal. Revista Internacional de Biología de Poblaciones, 2(2), 13-28.

Daniels, V. (1981). Manganese-containing stains on excavated pottery sherds. Masca Journal, 1, 230-231.

Dembo, A., \& Imbelloni, J. (1938). Deformaciones intencionales del cráneo de carácter étnico. Buenos Aires: José Anesi.

DeNiro, M. J. (1985). Postmortem preservation and alteration of in vivo bone collagen isotope ratios in relation to paleodietary reconstruction. Nature, 317, 806-809.

Favier Dubois, C. M., \& Scartascini, F. (2012). Intensive fishery scenarios on the North Patagonian coast (Río Negro, Argentina) during the Mid-Holocene. Quaternary International, 256(1), 62-70.

Favier Dubois, C. M., García Guraieb, S., Borella, F. \& Mariano, C. (2007). Primeros avances acerca del registro bioarqueológico de la costa rionegrina. Pacarina (volumen especial). Actas del XVI Congreso Nacional de Arqueología Argentina, (pp. 359-364). San Salvador de Jujuy.

Favier Dubois, C. M., Borella, F., \& Tykot, R. (2009). Explorando tendencias temporales en el uso del espacio y los recursos marinos en el Golfo San Matías (Río Negro). En M. Salemme, F. Santiago, M. Álvarez, E. Piana, M. Vázquez \& E. Mansur (Eds.), Arqueología de la Patagonia: una mirada desde el último confín, (pp. 985-997). Ushuaia: Editorial Utopías.

Fisher, A., \& Nacuzzi, L. R. (1992). La destrucción sistemática del paisaje y de los sitios arqueológicos. El caso del Valle de Viedma. Arqueología, 2, 189-229.

Flandes, L. B. (1978). Univariate and multivariate methods of sexing the sacrum. American Journal of Physical Anthropology, 49, 103-110.

Flensborg, G., Martínez, G., González, M., \& Bayala, P. (2011). Revisión de los restos óseos humanos del sitio La Petrona (transición pampeano-patagónica oriental, Argentina). Magallania, 39(1), 179-191.

Flensborg, G., Bayala, P., \& Martínez, G. (2017). Prácticas funerarias. Modalidades de inhumación y manejo de los cuerpos. En Martínez, G. (Ed.), Arqueología de cazadores-recolectores del curso inferior del Río Colorado (provincia de Buenos Aires, Argentina). Aportes al conocimiento de las ocupaciones humanas Pampeano-Patagónicas, (pp. 175-191). Olavarría: Serie Monográfica INCUAPA.

Flensborg, G., Martínez, G., \& Tessone, A. (2018). First approach to the paleodiet of hunter-gatherers through stable isotopes ( $\delta 13 \mathrm{C}$ and $\delta 15 \mathrm{~N}$ ) in the eastern PampaPatagonia transition during the Middle Holocene. Journal of Archaeological Science, 17, 571-580.

García Guraieb, S., Mariano, C., \& Favier Dubois, C. M. (2010). El buque sur: un entierro primario múltiple de 2300 años en la costa del Golfo San Matías, Río Negro, Argentina. Magallania, 38(1), 137-148.

Gifford-González, D. P. (1981). Taphonomy and Paleoecology: A Critical Review of Archaeology's Sister Disciplines. En Schiffer, M. B. (Ed.), Advances in Archaeological Method and Theory, (pp. 365-438). New York: Academic Press.

González, M. E. (2007). Estudios de interés tafonómico en los restos óseos humanos de laguna Tres Reyes 1 (Partido de Adolfo Gonzáles Chávez, provincia de Buenos Aires). Intersecciones en Antropología, 8, 215-233.

González, M. E. (2012). Procesos de formación en el registro bioarqueológico de la sub-región Pampa Húmeda y área ecotonal Pampa-Patagonia. Tesis Doctoral Inédita. Facultad de Ciencias Sociales, Universidad Nacional del Centro de la Provincia de Buenos Aires, Olavarría.

González-José, R. (2003). El poblamiento de la Patagonia. Análisis de la variación craneofacial en el contexto del poblamiento Americano. Tesis Doctoral Inédita. Universidad de Barcelona. Barcelona. España.

Gordón, F. (2011). Dinámica Poblacional, Conflicto y Violencia en el Norte de Patagonia durante el Holoceno Tardio: un Estudio Arqueológico. Tesis Doctoral Inédita. Buenos Aires: Universidad Nacional de La Plata.

Grayson, D. K. (1984). Quantitative Zooarchaeology: Topics in the Analysis of Archaeological Faunas. Orlando: Academic Press.

Gutiérrez, M. (2004). Análisis tafonómicos en el área Interserrana (Provincia de Buenos Aires). Tesis Doctoral Inédita. Facultad de Ciencias Naturales y Museo, Universidad Nacional de La Plata, La Plata.

Imbelloni, J. (1924-1925). Deformaciones intencionales del cráneo en Sud América. Revista del Museo de La Plata, 28, 329-407. 
Klein, R. G., \& Cruz-Uribe, K. (1984). The Analysis of Animal Bones from Archaeological Sites. Chicago: University of Chicago Press.

Krogman, W. (1962). The human skeleton in forensic medicine. Illinois: Charles C. Thomas.

Lista, R. [1880] (1975). Mis exploraciones y descubrimientos en la Patagonia (1877-1880). Buenos Aires: Marymar.

López-González, F., Grandal-d'Anglade, A., \& Vidal-Romaní, J. R. (2006). Deciphering bone depositional sequences in caves through the study of manganese coatings. Journal of Archaeological Science, 33, 707-717.

Lyman, R. L. (1994). Vertebrate Taphonomy. Cambridge Manuals in Archaeology. Cambridge: Cambridge University Press.

Lyman, R. L., \& Fox, G. L. (1989). A critical evaluation of bone weathering as an indication of bone assemblage formation. Journal of Archaeological Science, 16, 293-317.

Martin, F. M. (2002). Carnívoros y huesos humanos de FuegoPatagonia. Aporte desde la Tafonomía Forense. Tesis de Licenciatura Inédita. Facultad de Filosofía y Letras, Universidad de Buenos Aires.

Mariano, C. (2011). Prácticas mortuorias y registro bioarqueológico en la costa rionegrina del Golfo San Matías, Argentina. Intersecciones en Antropología, 12, 17-30.

Martínez, G. (2010). Entierros humanos en lugares sagrados y domésticos durante el Holoceno tardío: el registro bioarqueológico del curso inferior del río Colorado (provincia de Buenos Aires, Argentina). Werkén, 13, 145-161.

Martínez, G., \& Flensborg, G. (2018). Nuevos contextos funerarios en la transición pampeano Patagónica oriental (Argentina). Aportes al Holoceno Medio y Tardío Inicial. Chungará. Revista de Antropología Chilena. En prensa.

Martínez, G., Flensborg, G., \& Bayala, P. (2012). Human corpse manipulation and the body as symbol: A case study from the Eastern Pampa-Patagonia transition (Argentina) during the Final Late Holocene. Journal of Anthropological Archaeology, 31, 215-226.

Menéndez, L. (2010). Patologías bucales en cráneos humanos del noreste de Patagonia: Tendencias temporales en la dieta y estado de salud de las poblaciones del Holocenotardío del valle inferior del río Negro. Magallania, 38, 115-126.

Moldes de Entraigas, B. (1983). Arqueología y etnohistoria del bajo curso del Río Negro. Presencia hispánica en la arqueología Argentina, 2, 877-893.
Moreno, F. P. (1874). Description des cimetières et paraderos prehistoriques de Patagonie. Revue d'Antropologie, 3, 72-90.

Owings Webb, P., \& Suchey, J. (1985). Ephiphyseal union of the anterior iliac crest and medial clavicle in a modern multiracial sample of American males and females. American Journal of Physical Anthropology, 68, 457-466.

Pérez, S. I. (2006). El poblamiento holocénico del Sudeste de la Región Pampeana: un estudio de Morfometría geométrica craneofacial. Tesis Doctoral inédita, Universidad Nacional de La Plata.

Pérez, S. I., Cardillo, M., \& González, P. N. (2009). Artificial Cranial Deformations in South America: A quantitative approach to cultural variation. En G. López \& M. Cardillo (Eds.), Teoría, Métodos y Casos de Estudio en Arqueología Evolutiva, (pp. 87-107). Buenos Aires: Colección de Complejidad Humana, Editorial.

Phenice, T. (1969). A newly developed visual method of sexing in the os pubis. American Journal of Physical Anthropology, 30, 297-301.

Prates, L., \& Di Prado, V. (2013). Sitios con entierros humanos y ocupaciones residenciales en la cuenca del rio Negro (Norpatagonia, Argentina). Diacronia y multicausalidad. Latin American Antiquity, 24(4), 451-466.

Sanguinetti de Bórmida, A. (1999). Proyecto Nordpatagonia: Arqueología de la costa septentrional. Anales de la Academia Nacional de Ciencias, X, 3-35.

Sardi, M. L. (2002). Diferenciación craneofacial en aborígenes de la Patagonia y su relación con grupos americanos y extraamericanos. Tesis doctoral Inédita. Facultad de Ciencias Naturales y Museo, Universidad Nacional de La Plata.

Scartascini, F., \& Alberti, J. (2011). Rescate arqueológico Loteo Silva, Viedma, Rio Negro. Informe técnico para la Secretaria de Cultura de la Província de Rio Negro.

Serna, A., \& Romano, V. (2018). Rescates bioarqueológicos en el valle medio del río Negro (provincia de Río Negro): el potencial informativo del registro altamente perturbado. Revista Argentina de Antropología Biológica, 20(2), doi:10.17139/raab.2018.0020.02.03.

Serna, A., Prates, L., Flensborg, G., Martínez, G., Favier Dubois, C. M., \& Perez, S. I. (2018). Does the shape make a difference? Evaluating the ethnic role of cranial modification in Pampa-Patagonia region (Argentina) during the late Holocene. Archaeological and Anthropological Sciences, en prensa. ISSN 1866 9565 (Online).

Todd, L. \& Frison, G. (1992). Reassembly of bison skeletons 
from the Horner Site: a study in anatomical reffiting. En J. Hofman \& J. Enloe, J. (Eds.), Piecing Together the
Past: Applications of Reffiting Studies in Archaeology 578 (pp. 63-82). Oxford: BAR International Series. 